\title{
Correlation function of the Schur process with a fixed final partition
}

\author{
T. Imamura ${ }^{*}$ and T. Sasamoto ${ }^{\dagger}$ \\ * Institute of Industrial Science, University of Tokyo, \\ Komaba 4-6-1, Meguro-ku, Tokyo 153-8505, Japan \\ ${ }^{\dagger}$ Department of Mathematics and Informatics, \\ Chiba University, \\ Yayoi-cho 1-33, Inage-ku, Chiba 263-8522, Japan
}

\begin{abstract}
We consider a generalization of the Schur process in which a partition evolves from the empty partition into an arbitrary fixed final partition. We obtain a double integral representation of the correlation kernel. For a special final partition with only one row, the edge scaling limit is also discussed by the use of the saddle point analysis. If we appropriately scale the length of the row, the limiting correlation kernel changes from the extended Airy kernel.
\end{abstract}

*e-mail: timamura@iis.u-tokyo.ac.jp

${ }^{\dagger}$ e-mail: sasamoto@math.s.chiba-u.ac.jp 


\section{Introduction}

Recently in nonequilibrium stochastic models which belong to the one-dimensional KardarParisi-Zhang(KPZ) universality class [1], remarkable progress has been made on the understanding of the scaling function. The exact form of the function is obtained and its relation to the random matrix theory is revealed [2, 3, 4, 5, 6]. The common mathematical structure among many of these models is that certain "correlation functions" can be expressed as a determinant of a correlation kernel. This structure makes it possible to exactly analyze the function. The process having the structure is called determinantal process.

The Schur process, which was introduced in [7], is the typical process of the determinantal processes. Let $\lambda$ be a partition, i.e. $\lambda=\left(\lambda_{1}, \lambda_{2}, \cdots\right)$ where $\lambda_{i} \in\{0,1,2, \cdots\}$ and $\lambda_{1} \geq \lambda_{2} \geq \cdots$. For a set of partitions $\left\{\lambda^{(1)}, \lambda^{(2)}, \cdots, \lambda^{(4 N-1)}\right\}$, the measure is defined as

$$
s_{\lambda^{(1)}}\left(a^{(1)}\right)\left(\prod_{j=1}^{2 N-1} s_{\lambda^{(2 j-1)} / \lambda^{(2 j)}}\left(a^{(2 j)}\right) s_{\lambda^{(2 j+1)} / \lambda^{(2 j)}}\left(a^{(2 j+1)}\right)\right) s_{\lambda^{(4 N-1)}}\left(a^{(4 N)}\right),
$$

where $s_{\lambda / \mu}\left(a^{(i)}\right)$ is the skew Schur function of shape $\lambda / \mu$ in the variables $a^{(i)}=\left(a_{1}^{(i)}, a_{2}^{(i)}, \cdots\right)$. (For the definition of the Schur function, see (2.10).) Like the equation above, probability measures described by the Schur function appear in various fields in statistical mechanics. It has been known that the Schur function is useful for studying the problems of manybody nonintersecting random walk and Brownian motion due to its determinant structure and the relation to the combinatorics of the Young diagram [8, 9, 10, 11, 12, 13]. Furthermore the weight (1.1) and its variant appear in analyses of various nonequilibrium processes such as the polynuclear growth(PNG) model [14], melting in three-dimensional crystal [15], random tiling process [16], asymmetric exclusion process [17] and so on.

In order to visualize this model, we explain many-body random walk interpretation of the process introduced in [7]. At $t=0$ and $t=4 N$, the random walker labeled $i(i=$ $1,2, \cdots)$ is on the position $1-i$ on one dimensional lattice $\mathbb{Z}=\{\cdots,-2,-1,0,1,2, \cdots\}$. We regard $\lambda_{i}^{(n)}-i+1$ as the position of the $i$ th walker, at time $n$. Here $\lambda_{i}^{(n)}$ is the $i$ th element of the partition $\lambda^{(n)}=\left(\lambda_{1}^{(n)}, \lambda_{2}^{(n)}, \cdots, 0,0, \cdots\right)$. On the other hand, the Schur function $s_{\lambda^{(n+1)} / \lambda^{(n)}}\left(a^{(n)}\right)$ is interpreted as the transition weight of the walkers from the position $\left\{\lambda_{i}^{(n)}-i+1\right\}_{i=1,2, \cdots}$ at time $n$ to $\left\{\lambda_{i}^{(n+1)}-i+1\right\}_{i=1,2, \cdots}$ at time $n+1$. Note that the variables $a^{(n)}$ characterize the transition weight at each time step.

Due to the property $\lambda_{1}^{(n)}>\lambda_{2}^{(n)}-1>\cdots>\lambda_{i}^{(n)}-i+1>\lambda_{i+1}^{(n)}-i>\cdots$, the walkers do not intersect. Note that the transition weight (the Schur function) has the Slater determinant like structure (2.10). Thus, this nonintersecting random walk has the free fermionic feature and we find that (1.1) describes the (free fermionic) model of nonintersecting random walkers which depart from the positions $(0,-1,-2, \cdots)$ at time 0 and arrive at the same positions at time $4 N$ by way of the positions $\left\{\lambda_{i}^{(n)}-i+1\right\}_{i=1,2, \ldots}$ at time $n=1,2, \cdots, 4 N-1$, as depicted in Fig. 1 .

This type of nonintersecting random walk is closely related to the PNG model. In [14, 18], the authors introduced the same type of the random walk model called the multilayer 
PNG model in order to discuss the equal-time multipoint correlations of height fluctuations. In this model, the position of the first walker $\lambda_{1}^{(n)}$ at time $n,(n=1, \cdots, 4 N)$ represents the height at position $n$ and at some fixed time. Note that in order to find the dynamics of the first walker, we need the information about the correlation among the first walker and the other walkers. Thus, we focus on the correlation function (see (2.4)) of the Schur process in this paper. We are also interested in seeing how the initial or final configurations of the walkers affect the dynamical behavior. In the study of the PNG model with an external source [19, 20, 21], it has been known that the property of the height fluctuation changes if the external source is larger than a critical value. As we discussed in [21], the external source in the PNG model may be related to the initial or final configurations of the nonintersecting random walk model.

In order to discuss the problem, we investigate the following process in this paper:

$$
s_{\lambda^{(1)}}\left(a^{(1)}\right)\left(\prod_{j=1}^{2 N-1} s_{\lambda^{(2 j-1)} / \lambda^{(2 j)}}\left(a^{(2 j)}\right) s_{\lambda^{(2 j+1)} / \lambda^{(2 j)}}\left(a^{(2 j+1)}\right)\right) s_{\lambda^{(4 N-1)} / \mu^{(4 N)}}\left(a^{(4 N)}\right) .
$$

Here the partition $\mu^{(4 N)}=\left(\mu_{1}^{(4 N)}, \mu_{2}^{(4 N)}, \cdots, \mu_{n}^{(4 N)}, 0,0, \cdots\right)$ is arbitrary but fixed. Notice that the weight (1.2) is reduced to (1.1) if we choose $\mu^{(4 N)}=\phi:=(0,0, \cdots)$. In the interpretation of nonintersecting random walk, the partition $\mu^{(4 N)}$ corresponds to the final configuration $\left\{\mu_{i}^{(4 N)}-i+1\right\}_{i=1,2, \ldots}$. Thus, the weight (1.2) is a natural extension of the Schur process. Fig. 2 illustrates the situation. In this paper, we obtain the double integral formula of the correlation kernel of the (dynamical) correlation function (see (2.5)). Furthermore, by the use of the formula, we discuss the asymptotic limit of the correlation function. It will be shown that if we appropriately scale the partition $\mu^{(4 N)}$, the scaling limit changes from case (1.1).

In [7, Okounkov and Reshetikhin originally derived determinant structure of the correlation function and the integral representation of the correlation kernel in the Schur process (1.1) by the method of using the fermion operators. This method was first developed in their previous article [22]. In [14], Johansson considered the height fluctuation property of the (multilayer) PNG model which is essentially the same as process (1.1) and discussed the derivation of the integral formula by using a property of a Toeplitz matrix. Furthermore, the edge scaling limit(see Sec. 2.3) of the correlation kernel is also obtained. A simple linear algebraic proof of the integral formula along the approach in [14] and its Pfaffian analog were discussed by Borodin and Rains [23. In the noncolliding Brownian motion, which we can regard as the continuum version of Fig.1, the correlation function and its asymptotic form have been discussed in the similar situation to Fig. 1 where Brownian particles start and end at one point. It is found that the process is closely related to the random matrix theory and orthogonal polynomials [12, 24, 25]. The connection between the dynamics of the first particle and Painlevé equation is also revealed [26, 27].

In case (1.2), on the other hand, the asymptotics of the correlation function has not been discussed yet since an integral representation of the correlation kernel has not been obtained. In this paper, we discuss the generalization of the method in [14] in order to obtain the integral formula for the correlation kernel in process (1.2) although we could 
consider the generalization of the methods in [7, 23]. In the similar situation of noncolliding Brownian motion where the particles start from one initial point and converge to fixed final points at the end, it is revealed that the process is related to the random matrix model with external source and due to the properties of multiple orthogonal polynomials, its asymptotic limit can also be obtained [28, 21. Recently a partial differential equation describing the dynamics of the first particle is also obtained in this situation [29]. However, process (1.2) includes the parameters $a^{(i)}$ by which we can change the transition weight every time step. Therefore, (1.2) is a more general process in the sense that it includes both $a^{(i)}$ describing the temporary inhomogeneity and boundary parameter $\mu^{(4 N)}$.

This paper is arranged as follows. In Sec. 2, we discuss the background of this study especially the result about the process defined by products of determinant [14] and give our main results: the double integral formula of the correlation kernel (Theorem 2.1) and its edge scaling limit (Theorem 2.2). The double integral formula of the correlation kernel is derived in Sec. 3, In Sec. 4, we discuss the edge scaling limit of the correlation kernel applying the saddle point method to the integral formula. The Concluding remark is given in Sec. 5 .

\section{Correlation function}

\subsection{Determinant representation of correlation function}

The determinantal structure of correlation function has been derived for various stochastic processes such as the Schur process (1.1) [7] and random matrices [30]. For later discussion, we consider a class of general measures which contains the above measures and describe the result obtained in [14]. This is obtained by generalizing the discussion about the random matrix theory in 31 .

For the set $\left\{x^{(i)}\right\}_{i=1, \cdots, 4 N-1}$, where $x^{(i)}=\left(x_{1}^{(i)}, x_{2}^{(i)}, \cdots, x_{M}^{(i)}\right)$, we consider the weight defined by the products of determinants,

$$
w_{M, 4 N}\left(\left\{x^{(i)}\right\}_{i=1, \cdots, 4 N-1}\right)=\prod_{r=0}^{4 N-1} \operatorname{det}\left(\phi_{r, r+1}\left(x_{i}^{(r)}, x_{j}^{(r+1)}\right)\right)_{i, j=1}^{M},
$$

where $\left\{\phi_{r, r+1}(x, y)\right\}_{r=1, \cdots, 4 N}$ are some functions on $\mathbb{Z}^{2}$ and we fix $x^{(0)}$ and $x^{(4 N)}$ such that

$$
x_{1}^{(k)}>x_{2}^{(k)}>\cdots>x_{M}^{(k)}, \quad k=0,4 N .
$$

(In [14, some condition is assumed for the function $\phi_{r, r+1}(x, y)$ such that all objects that appear in the discussion converge.)

By using Lindström-Gessel-Viennot's method [32, 33] (see also [34]), we find that under condition (2.2), the weights assigned for all configurations but those satisfying

$$
x_{1}^{(i)}>x_{2}^{(i)}>\cdots>x_{M}^{(i)}
$$


vanish. Namely, when we interpret $x_{i}^{(j)}$ as the position of the particle labeled $i$ at time $j$ as in the previous section, the weight does not vanish only in the case where the particles do not intersect.

The correlation function of the measure (2.1) $R\left(x_{1}^{(1)}, \cdots, x_{k_{1}}^{(1)}, \cdots, x_{1}^{(4 N-1)}, \cdots, x_{k_{4 N-1}}^{(4 N-1)}\right)$ is defined as

$$
\begin{aligned}
R\left(x_{1}^{(1)}, \cdots, x_{k_{1}}^{(1)}, \cdots, x_{1}^{(4 N-1)}, \cdots,\right. & \left.x_{k_{4 N-1}}^{(4 N-1)}\right) \\
& =\frac{1}{Z}\left(\prod_{i=1}^{4 N-1} \sum_{x_{k_{i}+1}^{(i)}, \cdots, x_{M}^{(i)}=-\infty}^{\infty}\right) w_{M, 4 N}\left(\left\{x^{(i)}\right\}_{i=1, \cdots, 4 N-1}\right),
\end{aligned}
$$

where $Z=\prod_{i=1}^{4 N-1} \sum_{x_{1}^{(i)}, \cdots, x_{M}^{(i)}=-\infty}^{\infty} w_{M, 4 N}\left(\left\{x^{(i)}\right\}_{i=1, \cdots, 4 N-1}\right)$ is the normalization constant. In [14], Johansson showed that it can be represented as the determinant,

$$
\begin{aligned}
R\left(x_{1}^{(1)}, \cdots, x_{k_{1}}^{(1)}, \cdots, x_{1}^{(4 N-1)}, \cdots\right. & , x_{\left.k_{4 N-1}^{(4 N-1)}\right)} \\
& =\operatorname{det}\left(K\left(r, x_{j_{r}}^{(r)} ; s, x_{j_{s}}^{(s)}\right)\right)_{1 \leq r, s \leq 4 N-1,0 \leq j_{r} \leq k_{r}, 0 \leq j_{s} \leq k_{s}} .
\end{aligned}
$$

The correlation kernel $K\left(r_{1}, x_{1} ; r_{2}, x_{2}\right)$ is expressed as

$$
K\left(r_{1}, x_{1} ; r_{2}, x_{2}\right)=\tilde{K}\left(r_{1}, x_{1} ; r_{2}, x_{2}\right)-\phi_{r_{1}, r_{2}}\left(x_{1}, x_{2}\right)
$$

Here,

$$
\begin{aligned}
& \phi_{r, s}(x, y)= \begin{cases}\sum_{x_{r+1}=-\infty}^{\infty} \cdots \sum_{x_{s-1}=-\infty}^{\infty} \phi_{r, r+1}\left(x, x_{r+1}\right) \cdots \phi_{s-1, s}\left(x_{s-1}, y\right), & \text { for } r<s, \\
0, & \text { for } r \geq s,\end{cases} \\
& \tilde{K}\left(r_{1}, x_{1} ; r_{2}, x_{2}\right)=\sum_{i, j=1}^{M} \phi_{r_{1}, 4 N}\left(x_{1}, x_{i}^{(4 N)}\right)\left(\mathcal{A}^{-1}\right)_{i, j} \phi_{0, r_{2}}\left(x_{j}^{(0)}, x_{2}\right), \\
& \mathcal{A}_{i j}=\phi_{0,4 N}\left(x_{i}^{(0)}, x_{j}^{(4 N)}\right) .
\end{aligned}
$$

Hence, we find that in general class of measures, which is described by products of determinant, the correlation function is represented as the determinant.

Noticing the Jacobi-Trudi identity [35],

$$
s_{\lambda / \mu}(a)=\operatorname{det}\left(h_{\lambda_{i}-\mu_{j}+j-i}(a)\right),
$$

where $h_{k}(a)$ is the $k$ th complete symmetric polynomial in variables $a=\left(a_{1}, a_{2}, \cdots\right)$,

$$
h_{k}(a)=\sum_{i_{1} \leq \cdots \leq i_{k}} a_{i_{1}} \cdots a_{i_{k}}
$$


we easily find that the weight (1.2) has the form of (2.1), i.e., products of determinants under the following identification:

$$
\begin{aligned}
& x^{(0)}=(0,-1,-2, \cdots), \\
& x^{(4 N)}=\left(m_{1}, m_{2}, \cdots, m_{n},-n,-n-1, \cdots\right), \\
& m_{i}=\mu_{i}^{(4 N)}-i+1, \\
& x^{(j)}=\left(\lambda_{1}^{(j)}, \lambda_{2}^{(j)}-1, \lambda_{3}^{(j)}-2, \cdots\right), \text { for } j=1, \cdots, 4 N-1, \\
& \phi_{r, r+1}\left(x_{i}^{(r)}, x_{j}^{(r+1)}\right)= \begin{cases}h_{x_{i}^{(r+1)}-x_{j}^{(r)}}\left(a^{(r+1)}\right), & \text { for } r \text { even, } \\
h_{x_{i}^{(r)}-x_{j}^{(r+1)}}\left(a^{(r+1)}\right), & \text { for } r \text { odd. }\end{cases}
\end{aligned}
$$

Note that in (2.10), the rank of the determinant can be infinite (infinitely many walkers can move); hence, in this case, $M$ in (2.1) and (2.8) is infinity. Thus, under this identification (2.12)-(2.16) with $M \rightarrow \infty$, the result (2.5)-(2.9) is applicable to case (1.2).

\subsection{Double integral formula}

One of the purposes of this paper is to obtain a double integral formula of the correlation kernel in case (1.2), which is useful for the analysis of the scaling limit as $N$ goes to infinity. In case of the Schur process (1.1), the integral formula was first obtained in [7] by using the fermion operators. In this paper, however, for considering the case (1.2), we generalize the approach in [14] of calculating the inverse of the matrix $\mathcal{A}$ in (2.8) in case (2.12)-(2.16) . In the case of the Schur process (1.1), i.e., the case $\mu=\phi$ in (2.14), the matrix $\mathcal{A}$ is a Toeplitz matrix and its inverse $\mathcal{A}^{-1}$ can be estimated by using the Wiener-Hopf factorization [14]. However, in case (1.2), where $\mu^{(4 N)}$ is general, we cannot apply the method because $\mathcal{A}$ is not a Toeplitz matrix anymore, which is the main difficulty of this problem.

In this paper, we develop the method of estimating $\mathcal{A}^{-1}$ in the case of the weight (1.2) and give the double integral formula for the correlation kernel. In the following theorem, we consider the situation

$$
a^{(i)}=\left(a_{1}^{(i)}, a_{2}^{(i)}, \cdots, a_{p}^{(i)}, 0,0,0, \cdots\right)
$$

where $0<a_{j}^{(i)}<1$ for $i=1, \cdots, 4 N-1$ and $j=1, \cdots, p$. We can easily find that the conditions above ensures the convergence of the partition function $Z$ in (2.4).

The theorem is summarized as follows. The proof is given in the next section.

Theorem 2.1. In the case (2.12) -(2.17) and $r_{i}=2 u_{i}(i=1,2)$ in (2.6), $\tilde{K}\left(2 u_{1}, x_{1} ; 2 u_{2}, x_{2}\right)$ 
and $\phi_{2 u_{1}, 2 u_{2}}\left(x_{1}, x_{2}\right)$ in the correlation kernel (2.6) become

$$
\begin{aligned}
& \tilde{K}\left(2 u_{1}, x_{1} ; 2 u_{2}, x_{2}\right) \\
& =\frac{1}{(2 \pi i)^{2}} \int_{C_{r_{1}}} \frac{d z_{1}}{z_{1}^{1+x_{1}}} \int_{C_{r_{2}}} \frac{d z_{2}}{z_{2}^{1-x_{2}}} \prod_{m=1}^{p} \frac{\prod_{i=1}^{u_{1}}\left(1-a_{m}^{(2 i-1)} / z_{1}\right) \prod_{j=u_{2}+1}^{2 N}\left(1-a_{m}^{(2 j)} z_{2}\right)}{\prod_{k=u_{1}+1}^{2 N}\left(1-a_{m}^{(2 k)} z_{1}\right) \prod_{\ell=1}^{u_{2}}\left(1-a_{m}^{(2 \ell-1)} / z_{2}\right)} \\
& \times\left(\frac{z_{1}}{z_{1}-z_{2}}+\frac{1}{s_{\mu^{(4 N)}}(a)} \sum_{j=1}^{n} \sum_{\ell^{\prime}=0}^{m_{j}-1} h_{\ell^{\prime}}(a) z_{1}^{m_{j}-\ell^{\prime}} \sum_{b=1}^{n}(-1)^{j+b} z_{2}^{b-1} s_{\tilde{\mu}^{(j)} / \nu^{(b)}}(a)\right), \\
& \phi_{2 u_{1}, 2 u_{2}}\left(x_{1}, x_{2}\right)=\frac{1}{2 \pi i} \int_{C_{1}} \frac{d z}{z^{1+x_{2}-x_{1}}} \prod_{k=1}^{p} \prod_{i=u_{1}}^{u_{2}-1} \frac{1}{1-a_{k}^{(2 i+1)}} \prod_{i=u_{1}+1}^{u_{2}} \frac{1}{1-a_{k}^{(2 i)} / z},
\end{aligned}
$$

where $C_{r_{i}}$ denotes the contour with radius $r_{i}$ satisfying $r_{1}>r_{2}, h_{m}(a)$ is the $m$ th complete symmetric polynomial (2.11) in variables

$$
a=\left(a_{1}^{(1)}, a_{1}^{(3)}, a_{1}^{(5)}, \cdots, a_{1}^{(4 N-1)}, a_{2}^{(1)}, a_{2}^{(3)}, a_{2}^{(5)}, \cdots, a_{2}^{(4 N-1)}, \cdots, a_{p}^{(1)}, a_{p}^{(3)}, a_{p}^{(5)}, \cdots, a_{p}^{(4 N-1)}\right),
$$

and

$$
\begin{aligned}
& \mu^{(4 N)}=\left(\mu_{1}^{(4 N)}, \mu_{2}^{(4 N)}, \cdots, \mu_{n}^{(4 N)}\right)=\left(m_{1}, m_{2}+1, \cdots, m_{n}+n-1\right), \\
& \tilde{\mu}^{(j)}=\left(\mu_{1}^{(4 N)}+1, \cdots, \mu_{j-1}^{(4 N)}+1, \mu_{j+1}^{(4 N)}, \cdots, \mu_{n}^{(4 N)}\right), \\
& \nu^{(b)}=(\underbrace{1,1, \cdots, 1}_{b-1}, \underbrace{0, \cdots, 0}_{n-b+1}) .
\end{aligned}
$$

As was discussed in [14, 23], the condition (2.17) can be relaxed such that the product $\prod_{m=1}^{p} \frac{\prod_{i=1}^{u_{1}}\left(1-a_{m}^{(2 i-1)} / z_{1}\right) \prod_{j=u_{2}+1}^{2 N}\left(1-a_{m}^{(2 j)} z_{2}\right)}{\prod_{k=u_{1}+1}^{2 N}\left(1-a_{m}^{(2 k)} z_{1}\right) \prod_{\ell=1}^{u_{2}}\left(1-a_{m}^{(2 \ell-1)} / z_{2}\right)}$ converges. Note that in case $m_{i}=0$ (the Schur process), the second term in (2.18) vanishes and it reduces to the integral formula in [7].

\subsection{Edge scaling limit}

The benefit of the representation (2.18) is that we can take an asymptotic limit of the correlation kernel. In this paper, we consider the edge scaling limit of the correlation kernel in the special situation

$$
\begin{aligned}
& x^{(4 N)}=(m,-1,-2, \cdots), \\
& a^{(1)}=\cdots=a^{(4 N)}=(\alpha, 0,0, \cdots) .
\end{aligned}
$$

In (2.24) with $m=0$, the scaling limit of the correlation function was analyzed in [14] in order to study the height fluctuation of the PNG model. We discuss how the scaling behavior depends on the value of $m$. 
It has been known that in the case $m=0$, the trace of the first particle $x_{1}^{(t)}, \quad t=$ $0, \cdots, 4 N$, behaves asymptotically like

$$
\lim _{N \rightarrow \infty} \frac{x_{1}^{(t)}}{N}=A(t):=\frac{2 \alpha^{2}}{1-\alpha^{2}}+\frac{\alpha}{1-\alpha^{2}} \sqrt{4 t / N-(t / N)^{2}} .
$$

This represents a semi-circle centered at $t=2 N$. We focus our attention on the fluctuation of the walkers' positions $x_{i}, i=1,2, \cdots$, around the above limiting value at $t=2 N$. The scaling limit is called the edge scaling limit. Precisely, $x_{i}$ and $2 u_{i}$ in (2.18) and (2.19) are scaled as follows:

$$
\begin{aligned}
& x_{i}=A\left(2 u_{i}\right) N+D N^{1 / 3} \xi_{i}, \\
& 2 u_{i}=2 N+2 C N^{2 / 3} \tau_{i},
\end{aligned}
$$

where $A(t)$ is defined in (2.26) and

$$
D=\frac{\alpha^{1 / 3}}{1-\alpha^{2}}(1+\alpha)^{4 / 3}, \quad C=\frac{(1+\alpha)^{2 / 3}}{\alpha^{1 / 3}} .
$$

The exponent $1 / 3$ (resp.2/3) in (2.27) (resp. (2.28) ) characterizes the one-dimensional KPZ universality [2, 3, 4, 5, 6]. We also scale $m$ in (2.24) as

$$
m=A(2 N) N+B N^{2 / 3} \omega
$$

where

$$
B=\frac{2 \alpha^{2 / 3}}{(1-\alpha)(1+\alpha)^{1 / 3}}
$$

Our result of the scaling limit is summarized as follows. The proof will be given in Section 4 .

Theorem 2.2. In the situation (2.24)-(2.31), the correlation kernel has the following scaling limit

$$
\begin{aligned}
& \lim _{N \rightarrow \infty} K\left(2 u_{1}, x_{1} ; 2 u_{2}, x_{2}\right) \frac{D N^{1 / 3}}{P} \\
& = \begin{cases}\mathcal{K}_{2}\left(\tau_{1}, \xi_{1} ; \tau_{2}, \xi_{2}\right)+\int_{0}^{\infty} d \lambda e^{\lambda\left(\tau_{1}+\omega\right)} \operatorname{Ai}\left(\xi_{1}-\lambda\right) \operatorname{Ai}\left(\xi_{2}\right), & \tau_{1}+\omega \leq 0, \\
\mathcal{K}_{2}\left(\tau_{1}, \xi_{1} ; \tau_{2}, \xi_{2}\right)-\int_{0}^{\infty} d \lambda e^{-\lambda\left(\tau_{1}+\omega\right)} \operatorname{Ai}\left(\xi_{1}+\lambda\right) \operatorname{Ai}\left(\xi_{2}\right) & \\
+\operatorname{Ai}\left(\xi_{2}\right) \exp \left(-\frac{\left(\tau_{1}+\omega\right)^{3}}{3}+\xi_{1}\left(\tau_{1}+\omega\right)\right), & \tau_{1}+\omega>0,\end{cases}
\end{aligned}
$$

where $D$ is defined in (2.29), $P$ (see (4.4)) is a factor which does not contribute the determinant in (2.5), and

$$
\mathcal{K}_{2}\left(\tau_{1}, \xi_{1} ; \tau_{2}, \xi_{2}\right)= \begin{cases}\int_{0}^{\infty} d \nu e^{-\nu\left(\tau_{1}-\tau_{2}\right)} \operatorname{Ai}\left(\xi_{1}+\nu\right) \operatorname{Ai}\left(\xi_{2}+\nu\right), & \tau_{1} \geq \tau_{2} \\ -\int_{-\infty}^{0} d \nu e^{-\nu\left(\tau_{1}-\tau_{2}\right)} \operatorname{Ai}\left(\xi_{1}+\nu\right) \operatorname{Ai}\left(\xi_{2}+\nu\right), & \tau_{1}<\tau_{2}\end{cases}
$$


When $m=0$ in (2.24), which corresponds to case $(1.1)\left(\mu^{(4 N)}=\phi\right)$, only the term $\mathcal{K}_{2}\left(\tau_{1}, \xi_{1} ; \tau_{2}, \xi_{2}\right)$ is left since this case corresponds to $\omega \rightarrow-\infty$. The correlation kernel $\mathcal{K}_{2}\left(\tau_{1}, \xi_{1} ; \tau_{2}, \xi_{2}\right)$ is called the extended Airy kernel, which appeared in the random matrix theory [36, 37, 38, 39] and the PNG model [14, 18]. Recently, the correlation kernel (2.32) has also appeared in various fields such as the random matrix with external source (including the noncolliding Brownian motion with pinned initial or final condition) [21, 29], the PNG model with an external source [40, 20], statistics [41], and so on.

\section{Integral representation for the correlation kernel}

In this section, we give the proof of Theorem 2.1, First, we represent $\phi_{r, r+1}(x, y)(2.16)$ in terms of the vertex operators defined in (A.5). The analysis of the Schur process using the vertex operators were developed in [7, 22]. However, note that in our analysis, we adopt the one-particle basis whereas in [7, 22], infinitely-many particle state is chosen as a basis. Basic properties of the vertex operators and symmetric functions are summarized in Appendix A.

First, we prove (2.19). From (2.10) and (A.13), we have

$$
\phi_{r, r+1}(x, y)= \begin{cases}\left\langle y\left|H_{+}\left(s^{(r+1)}\right)\right| x\right\rangle, & \text { for } r \text { even } \\ \left\langle y\left|H_{-}\left(s^{(r+1)}\right)\right| x\right\rangle, & \text { for } r \text { odd }\end{cases}
$$

Here, the parameter $s^{(j)}=\left(s_{1}^{(j)}, s_{2}^{(j)}, \cdots\right)$ is connected to $a^{(j)}$ in (1.2) as

$$
s_{k}^{(j)}=\frac{1}{k} \sum_{i=1}^{p} a_{i}^{(j) k}
$$

By using (3.1) and (A.9), we can express $\phi_{2 u_{1}, 2 u_{2}}$ (2.7) as

$$
\phi_{2 u_{1}, 2 u_{2}}\left(x_{1}, x_{2}\right)=\left\langle x_{2}\left|\prod_{i=u_{1}}^{u_{2}-1} H_{+}\left(s^{(2 i+1)}\right) \prod_{j=u_{1}+1}^{u_{2}} H_{-}\left(s^{(2 j)}\right)\right| x_{1}\right\rangle .
$$

By applying (A.6) and (A.7) to this equation, we easily get (2.19). 
Next, we consider the integral form of $\tilde{K}$. Eq. (2.8) is written as

$$
\begin{aligned}
\tilde{K} & \left(2 u_{1}, x_{1} ; 2 u_{2}, x_{2}\right) \\
= & \frac{1}{(2 \pi i)^{2}} \int_{C_{r_{1}}} \frac{d z_{1}}{z_{1}^{1+x_{1}}} \int_{C_{r_{2}}} \frac{d z_{2}}{z_{2}^{1-x_{2}}} \frac{z_{1}}{z_{2}} \sum_{i, j=1}^{\infty} z_{1}^{x_{i}^{(4 N)}-1} \mathcal{A}_{i j}^{-1} z_{2}^{x_{j}^{(0)}+1}\left(\sum_{a=-\infty}^{\infty} \phi_{2 u_{1}, 4 N}\left(a, x_{i}^{(4 N)}\right) z_{1}^{a-x_{i}^{(4 N)}}\right) \\
& \times\left(\sum_{b=-\infty}^{\infty} \phi_{0,2 u_{2}}\left(x_{j}^{(0)}, b\right) z_{2}^{x_{j}^{(0)}-b}\right) \\
= & \frac{1}{(2 \pi i)^{2}} \int_{C_{r_{1}}} \frac{d z_{1}}{z_{1}^{1+x_{1}}} \int_{C_{r_{2}}} \frac{d z_{2}}{z_{2}^{1-x_{2}}} \frac{z_{1}}{z_{2}} \sum_{i, j=1}^{\infty} z_{1}^{x_{i}^{(4 N)}-1} \mathcal{A}_{i j}^{-1} z_{2}^{j} \\
& \times \prod_{i=u_{1}}^{2 N-1} \gamma\left(z_{1}^{-1}, s^{(2 i+1)}\right) \prod_{j=u_{1}+1}^{2 N} \gamma\left(z_{1}, s^{(2 j)}\right) \prod_{k=0}^{u_{2}-1} \gamma\left(z_{2}^{-1}, s^{(2 k+1)}\right) \prod_{\ell=1}^{u_{2}} \gamma\left(z_{2}, s^{(2 \ell)}\right) .
\end{aligned}
$$

Here, $\gamma(z, s)$ is defined in (A.12) and $C_{r_{i}}(i=1,2)$ denotes the contour centered at the origin anticlockwise with radius $r_{i}$ satisfying $r_{2}<r_{1}$. In the second equality, we use (3.3) and (A.6) $-($ A.8) .

Thus, what we have to do is to obtain an explicit form of the inverse of the (semi-infinite) matrix $\mathcal{A}(2.9)$ in case $(2.12)-(2.16)$ and calculate $\sum_{i, j=1}^{\infty} z_{1}^{x_{i}^{(4 N)}-1} \mathcal{A}_{i j}^{-1} z_{2}^{j}$. From (3.3), the matrix $\mathcal{A}$ in our case (2.12) -(2.16) can be expressed as

$$
\mathcal{A}_{i j}=\left\langle x_{j}\left|\Lambda_{+}(s) \Lambda_{-}\left(s^{\prime}\right)\right| 1-i\right\rangle,
$$

where

$$
\begin{aligned}
& x_{j}\left(=x_{j}^{(4 N)}\right)= \begin{cases}m_{j}, & \text { for } 1 \leq j \leq n, \\
1-j, & \text { for } n+1 \leq j,\end{cases} \\
& \Lambda_{+}(s)=\prod_{j=1}^{2 N} H_{+}\left(s^{(2 j-1)}\right), \quad \Lambda_{-}\left(s^{\prime}\right)=\prod_{j=1}^{2 N} H_{-}\left(s^{(2 j)}\right), \\
& s=\left(s^{(1)}, s^{(3)}, \cdots, s^{(4 N-1)}\right), \quad s^{\prime}=\left(s^{(2)}, s^{(4)}, \cdots, s^{(4 N)}\right) .
\end{aligned}
$$

In order to get $\mathcal{A}^{-1}$, we introduce the following matrix:

$$
\mathcal{A}_{i j}^{\prime}=\left\langle 1-j\left|\Lambda_{-}^{-1}\left(s^{\prime}\right) \mathbb{P}^{\prime} \Lambda_{+}^{-1}(s)\right| x_{i}\right\rangle,
$$

where $\mathbb{P}^{\prime}$ is a deformed projection operator

$$
\mathbb{P}^{\prime}=\sum_{j=1}^{\infty}|1-j\rangle\left\langle x_{j}\right| .
$$

Note that when $n=0$ in $(\underline{3.6}), \mathbb{P}^{\prime}$ becomes an ordinary projection operator $\mathbb{P}$ defined as

$$
\mathbb{P}=\sum_{j=1}^{\infty}|1-j\rangle\langle 1-j| .
$$


By the use of $\mathbb{P}^{\prime}$ and $\mathbb{P}$, we can express the product $\mathcal{A}^{\prime} \mathcal{A}$ as

$$
\left(\mathcal{A}^{\prime} \mathcal{A}\right)_{i k}=\left\langle x_{k}\left|\Lambda_{+}(s) \Lambda_{-}\left(s^{\prime}\right) \mathbb{P} \Lambda_{-}^{-1}\left(s^{\prime}\right) \mathbb{P}^{\prime} \Lambda_{+}^{-1}(s)\right| x_{i}\right\rangle .
$$

Let us focus on the term

$$
\mathbb{P} \Lambda_{-}^{-1}\left(s^{\prime}\right) \mathbb{P}^{\prime}=\sum_{p=1}^{\infty} \sum_{p^{\prime}=1}^{\infty}|1-p\rangle\left\langle 1-p\left|\Lambda_{-}^{-1}\left(s^{\prime}\right)\right| 1-p^{\prime}\right\rangle\left\langle x_{p^{\prime}}\right|
$$

Noticing $1-p^{\prime}<0$ and $<j\left|\Lambda_{-}^{-1}\left(s^{\prime}\right)\right| i>=0$ for $j>i$, which immediately follows from definition (3.7), we have

$$
\mathbb{P} \Lambda_{-}^{-1}\left(s^{\prime}\right) \mathbb{P}^{\prime}=\Lambda_{-}^{-1}\left(s^{\prime}\right) \mathbb{P}^{\prime} .
$$

Namely, we can change $\mathbb{P}$ into the identity operator in (3.13). Thus, we obtain

$$
\left(\mathcal{A}^{\prime} \mathcal{A}\right)_{i k}=\left\langle x_{k}\left|\Lambda_{+}(s) \mathbb{P}^{\prime} \Lambda_{+}^{-1}(s)\right| x_{i}\right\rangle
$$

Note that when $n=0$, one can easily find that $\mathbb{P}^{\prime}(=\mathbb{P})$ can also be changed into the identity operator in (3.15) from the similar discussion about (3.14). Thus, in the case $n=0, \mathcal{A}^{\prime} \mathcal{A}=1$ or, equivalently, $\mathcal{A}^{\prime}$ is nothing but $\mathcal{A}^{-1}$.

In the case of general $n$, we can represent the matrix $\mathcal{A}^{\prime} \mathcal{A}$ in terms of $c_{j-k}(s)$ and $d_{j-k}(s)$ defined in (A.14) and (A.15) respectively, i.e.,

$$
c_{j-k}(s)=\left\langle j\left|\Lambda_{+}(s)\right| k\right\rangle=\left\langle k\left|\Lambda_{-}(s)\right| j\right\rangle, \quad d_{j-k}(s)=\left\langle j\left|\Lambda_{+}^{-1}(s)\right| k\right\rangle=\left\langle k\left|\Lambda_{-}^{-1}(s)\right| j\right\rangle .
$$

By noting $c_{j-k}=d_{j-k}=0$ for $j-k<0$, we get

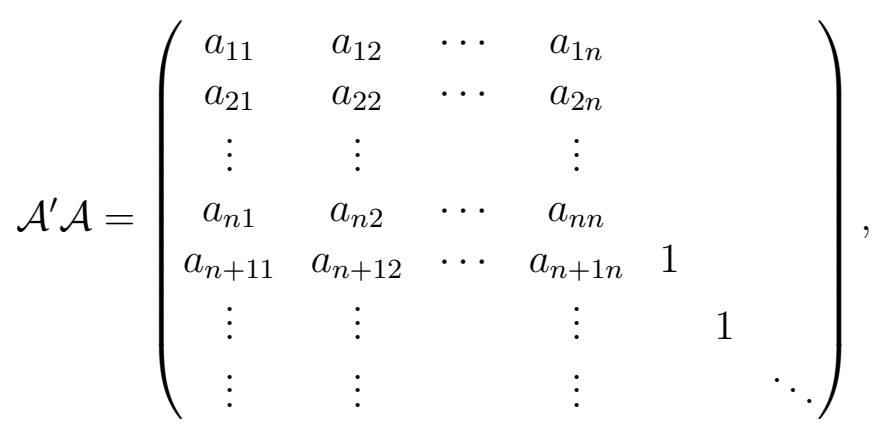

where the region after the $n+1$ th column is equivalent to the unit matrix and

$$
a_{i k}= \begin{cases}\sum_{j=1}^{i} d_{m_{j}-m_{i}}(s) c_{m_{k}+j-1}(s), & 1 \leq i \leq n, 1 \leq k \leq n \\ \sum_{j=n+1}^{i} c_{m_{k}+j-1}(s) d_{i-j}(s)+\sum_{j=1}^{n} d_{m_{j}+i-1}(s) c_{m_{k}+j-1}(s), & n+1 \leq i, 1 \leq k \leq n\end{cases}
$$


Here, $m_{i}$ is defined in (2.14). Note that the matrix $\mathcal{A}^{\prime} \mathcal{A}$ is almost the unit matrix. We easily find that the inverse of the matrix $\mathcal{A}^{\prime} \mathcal{A}$ has the following form:



By introducing

$$
\begin{aligned}
& \mathcal{A}^{\prime \prime}:=\left(a_{i j}\right)_{i, j=1}^{n}, \quad \tilde{\mathcal{A}}^{\prime \prime}{ }_{(j i)}: j i \text { cofactor of } \mathcal{A}^{\prime \prime}, \\
& \mathcal{C}=\left(c_{m_{j}+i-1}(s)\right)_{i, j=1}^{n},
\end{aligned}
$$

we find that the elements $b_{0}$ and $b_{i, j}$ can be expressed as

$$
\begin{aligned}
& b_{0}=\operatorname{det} \mathcal{A}^{\prime \prime}=\operatorname{det} \mathcal{C}, \\
& b_{i j}=\left\{\begin{array}{c}
\tilde{\mathcal{A}}^{\prime \prime}{ }_{(j i)}, \\
-\operatorname{det}\left(\begin{array}{cccc}
a_{11} & a_{12} & \ldots & a_{1 n} \\
\vdots & \vdots & & \vdots \\
a_{j-11} & a_{j-12} & \ldots & a_{j-1 n} \\
a_{i 1} & a_{i 2} & \ldots & a_{i n} \\
a_{j+11} & a_{j+12} & \ldots & a_{j+1 n} \\
\vdots & \vdots & & \vdots \\
a_{n 1} & a_{n 2} & \ldots & a_{n n}
\end{array}\right)=-\sum_{k=1}^{n} a_{i k} b_{k j}, \quad n+1 \leq i, 1 \leq j \leq n, 1 \leq j \leq n
\end{array}\right.
\end{aligned}
$$

Thus the inverse of the matrix $\mathcal{A}(2.9)$ is represented as

$$
\begin{aligned}
\mathcal{A}^{-1}= & \mathcal{B A}^{\prime} \\
& =\left(\begin{array}{ccc}
\frac{1}{b_{0}} \sum_{k=1}^{n} b_{1 k} \mathcal{A}_{k 1}^{\prime} & \frac{1}{b_{0}} \sum_{k=1}^{n} b_{1 k} \mathcal{A}_{k 2}^{\prime} & \ldots \\
\vdots & \vdots & \\
\frac{1}{b_{0}} \sum_{k=1}^{n} b_{n k} \mathcal{A}_{k 1}^{\prime} & \frac{1}{b_{0}} \sum_{k=1}^{n} b_{n k} \mathcal{A}_{k 2}^{\prime} & \ldots \\
\frac{1}{b_{0}} \sum_{k=1}^{n} b_{n+1 k} \mathcal{A}_{k 1}^{\prime}+\mathcal{A}_{n+1,1}^{\prime} & \frac{1}{b_{0}} \sum_{k=1}^{n} b_{n+1 k} \mathcal{A}_{k 2}^{\prime}+\mathcal{A}_{n+1,2}^{\prime} & \ldots \\
\frac{1}{b_{0}} \sum_{k=1}^{n} b_{n+2 k} \mathcal{A}_{k 1}^{\prime}+\mathcal{A}_{n+2,1}^{\prime} & \frac{1}{b_{0}} \sum_{k=1}^{n} b_{n+2 k} \mathcal{A}_{k 2}^{\prime}+\mathcal{A}_{n+2,2}^{\prime} & \ldots \\
\vdots & \vdots
\end{array}\right) .
\end{aligned}
$$


By using this expression for $\mathcal{A}^{-1}$, we can represent $\sum_{i, j=1}^{\infty} z_{1}^{x_{i}^{(4 N)}-1} \mathcal{A}_{i j}^{-1} z_{2}^{j}$ in (3.4) as

$$
\begin{aligned}
\sum_{i, j=1}^{\infty} z_{1}^{x_{i}-1}\left(\mathcal{A}^{-1}\right)_{i j} z_{2}^{j}= & \sum_{i=n+1}^{\infty} \sum_{j=1}^{\infty} z_{1}^{-i} \mathcal{A}_{i j}^{\prime} z_{2}^{j}+\frac{1}{b_{0}} \sum_{k=1}^{n} \sum_{i=n+1}^{\infty} z_{1}^{-i} b_{i k} \sum_{j=1}^{\infty} \mathcal{A}_{k j}^{\prime} z_{2}^{j} \\
& +\frac{1}{b_{0}} \sum_{k=1}^{n} \sum_{i=1}^{n} z_{1}^{m_{i}-1} b_{i k} \sum_{j=1}^{\infty} \mathcal{A}_{k j}^{\prime} z_{2}^{j} .
\end{aligned}
$$

After quite lengthy calculations of this equation, we obtain the following expression

$$
\begin{aligned}
\sum_{i, j=1}^{\infty} z_{1}^{x_{i}-1}\left(\mathcal{A}^{-1}\right)_{i j} z_{2}^{j}= & \left(\sum_{k=1}^{\infty}\left(\frac{z_{2}}{z_{1}}\right)^{k}+\frac{1}{\operatorname{det} \mathcal{C}} \sum_{j=1}^{n} \sum_{\ell^{\prime}=0}^{m_{j}-1} c_{\ell^{\prime}}(s) z_{1}^{m_{j}-1-\ell^{\prime}} \sum_{b=1}^{n} z_{2}^{b} \tilde{\mathcal{C}}_{(b j)}\right) \\
& \times \prod_{j=1}^{2 N} \gamma^{-1}\left(z_{1}^{-1}, s^{(2 j-1)}\right) \gamma^{-1}\left(z_{2}, s^{(2 j)}\right),
\end{aligned}
$$

where $\tilde{\mathcal{C}}_{(b j)}$ is the $b j$ cofactor of matrix $\mathcal{C}$. The proof of this equation is given in Appendix B. By noticing (A.13) and (A.14), we can rewrite $c_{\ell^{\prime}}(s), \operatorname{det} \mathcal{C}$, and $\tilde{\mathcal{C}}_{(b j)}$ as

$$
\begin{aligned}
& c_{\ell^{\prime}}(s)=h_{\ell^{\prime}}(a), \\
& \operatorname{det} \mathcal{C}=s_{\mu^{(4 N)}}(a), \\
& \tilde{\mathcal{C}}_{(b j)}=(-1)^{j+b} s_{\tilde{\mu}^{(j)} / \nu^{(b)}}(a),
\end{aligned}
$$

where $a, \mu^{(4 N)}, \tilde{\mu}^{(j)}$, and $\nu^{(b)}$ are defined in (2.20)-(2.23), respectively.

By substituting (3.26)-(3.29) into (3.4) and noting (3.2) and (3.12), we get the desired expression (2.18).

\section{Asymptotic analysis}

In this section, we give the proof of Theorem 2.2. We investigate the asymptotics of the correlation kernel by the use of the saddle point analysis.

In case (2.24) and (2.25), the correlation kernel defined in (2.18) and (2.19) reduces to

$$
\begin{aligned}
& K\left(2 u_{1}, x_{1} ; 2 u_{2}, x_{2}\right)=\tilde{K}\left(2 u_{1}, x_{1} ; 2 u_{2}, x_{2}\right)-\phi_{2 u_{1}, 2 u_{2}}\left(x_{1}, x_{2}\right) \\
& =\frac{1}{(2 \pi i)^{2}} \int_{C_{r_{1}}} \frac{d z_{1}}{z_{1}^{1+x_{1}}} \int_{C_{r_{2}}} \frac{d z_{2}}{z_{2}^{1-x_{2}}} \frac{z_{1}}{z_{1}-z_{2}} \frac{\left(1-\alpha / z_{1}\right)^{u_{1}}\left(1-\alpha z_{2}\right)^{2 N-u_{2}}}{\left(1-\alpha z_{1}\right)^{2 N-u_{1}}\left(1-\alpha / z_{2}\right)^{u_{2}}} \\
& +\frac{1}{2 \pi i} \int_{C_{1}} \frac{d z}{z^{1+x_{2}-x_{1}}}(1-\alpha z)^{u_{1}-u_{2}}(1-\alpha / z)^{u_{1}-u_{2}} \\
& +\sum_{j=1}^{m} \frac{h_{m-j}\left(\alpha^{2 N}\right)}{h_{m}\left(\alpha^{2 N}\right)} \frac{1}{2 \pi i} \int_{C_{1}} \frac{d z_{1}}{z_{1}^{1+x_{1}-j}} \frac{\left(1-\alpha / z_{1}\right)^{u_{1}}}{\left(1-\alpha z_{1}\right)^{2 N-u_{1}}} \times \frac{1}{2 \pi i} \int_{C_{1}} \frac{d z_{2}}{z_{2}^{1-x_{2}}} \frac{\left(1-\alpha z_{2}\right)^{2 N-u_{2}}}{\left(1-\alpha / z_{2}\right)^{u_{2}}}
\end{aligned}
$$


where $C_{r}$ denotes the contour enclosing the origin anticlockwise with radius $r$ and $h_{j}\left(\alpha^{2 N}\right)$ is the $j$ th complete symmetric polynomial (2.11) in variables

$$
\alpha^{2 N}=\underbrace{(\alpha, \alpha, \cdots, \alpha)}_{2 N} .
$$

In [14], it was shown that the first two terms of the correlation kernel above converge to the extended Airy kernel in the edge scaling limits (2.27) and (2.28),

$$
\begin{aligned}
& \lim _{N \rightarrow \infty} \frac{D N^{1 / 3}}{(2 \pi i)^{2}} \int_{C_{r_{1}}} \frac{d z_{1}}{z_{1}^{1+x_{1}}} \int_{C_{r_{2}}} \frac{d z_{2}}{z_{2}^{1-x_{2}}} \frac{z_{1}}{z_{1}-z_{2}} \frac{\left(1-\alpha / z_{1}\right)^{u_{1}}\left(1-\alpha z_{2}\right)^{2 N-u_{2}}}{\left(1-\alpha z_{1}\right)^{2 N-u_{1}}\left(1-\alpha / z_{2}\right)^{u_{2}}} \\
& +\frac{D N^{1 / 3}}{2 \pi i} \int_{C_{1}} \frac{d z}{z^{1+x_{2}-x_{1}}}(1-\alpha z)^{u_{1}-u_{2}}(1-\alpha / z)^{u_{1}-u_{2}}=P \times \mathcal{K}_{2}\left(\tau_{1}, \xi_{1} ; \tau_{2}, \xi_{2}\right),
\end{aligned}
$$

where $\mathcal{K}_{2}\left(\tau_{1}, \xi_{1} ; \tau_{2}, \xi_{2}\right)$ is defined in (2.33) and $P$ is a prefactor which does not contribute a determinant,

$$
P=(1-\alpha)^{\frac{2(1+\alpha)^{2 / 3} N^{2 / 3}\left(\tau_{1}-\tau_{2}\right)}{\alpha^{1 / 3}}} e^{\left(\tau_{1}^{3}-\tau_{2}^{3}\right) / 3+\xi_{2} \tau_{2}-\xi_{1} \tau_{1}} .
$$

Thus, we only consider the asymptotics of the last term,

$$
\sum_{j=1}^{m} \frac{h_{m-j}\left(\alpha^{2 N}\right)}{h_{m}\left(\alpha^{2 N}\right)} \psi_{1}\left(x_{1}-j\right) \psi_{2}\left(x_{2}\right)
$$

where

$$
\begin{aligned}
& \psi_{1}\left(x_{1}-j\right)=\frac{1}{2 \pi i} \int_{C_{1}} \frac{d z_{1}}{z_{1}^{1+x_{1}-j}} \frac{\left(1-\alpha / z_{1}\right)^{u_{1}}}{\left(1-\alpha z_{1}\right)^{2 N-u_{1}}}, \\
& \psi_{2}\left(x_{2}\right)=\frac{1}{2 \pi i} \int_{C_{1}} \frac{d z_{2}}{z_{2}^{1-x_{2}}} \frac{\left(1-\alpha z_{2}\right)^{2 N-u_{2}}}{\left(1-\alpha / z_{2}\right)^{u_{2}}}
\end{aligned}
$$

under the scaling (2.27)-(2.31). We scale $j$ as

$$
j=D N^{1 / 3} \lambda \text {. }
$$

Here, $D$ is defined in (2.29). This scaling makes a dominant contribution to the asymptotic limit of (4.5).

First, we consider the function $\psi_{1}\left(x_{1}-j\right)$. By substituting (2.28) into (4.6), we have

$$
\psi_{1}\left(x_{1}-j\right)=\frac{1}{2 \pi i} \int_{C_{1}} \frac{d z_{1}}{z_{1}^{1+x_{1}-j-\mu N}} \exp \left(N f_{\beta, \mu}\left(z_{1}\right)\right),
$$

where

$$
\begin{aligned}
& f_{\beta, \mu}(z)=(1+\beta) \log (z-\alpha)-(1-\beta) \log (1-\alpha z)-(\mu+1+\beta) \log z, \\
& \beta=C N^{-1 / 3} \tau_{1}
\end{aligned}
$$


and we fix $\mu$ as

$$
\mu=\frac{2 \alpha}{1-\alpha^{2}}\left(\alpha+\sqrt{1-\beta^{2}}\right)=A\left(2 u_{1}\right),
$$

where $A\left(2 u_{1}\right)$ is defined in (2.26). In (4.10), $C$ is defined in (2.29). We find that due to (4.11), two saddle points of the function $f_{\beta, \mu}(z)$ merge to the double saddle point $z_{c}(\beta)$,

$$
z_{c}(\beta)=\frac{\sqrt{1+\beta}+\alpha \sqrt{1-\beta}}{\sqrt{1-\beta}+\alpha \sqrt{1+\beta}} .
$$

We also have the relations

$$
\frac{f_{\beta, \mu}\left(z_{c}(\beta)\right)}{d z}=\frac{d^{2} f_{\beta, \mu}\left(z_{c}(\beta)\right)}{d z^{2}}=0, \frac{d^{3} f_{\beta, \mu}\left(z_{c}(\beta)\right)}{d z^{3}}=\frac{2 D^{3}}{z_{c}^{3}(\beta)} .
$$

Since the main contribution to the integral in (4.9) is given around $z \sim z_{c}(\beta), z_{1}$ may be transformed to

$$
z_{1}=z_{c}(\beta)\left(1-\frac{i w_{1}}{D N^{1 / 3}}\right) \sim 1+\frac{\tau_{1}-i w_{1}}{D N^{1 / 3}} .
$$

Then, we obtain the relations

$$
\begin{aligned}
\exp \left(N f_{\beta, \mu}\left(z_{1}\right)\right) & \sim \exp \left(N\left(f_{\beta, \mu}\left(z_{c}(\beta)\right)+\frac{f_{\beta, \mu}^{\prime \prime \prime}\left(z_{c}(\beta)\right)}{6}\left(z-z_{c}(\beta)\right)^{3}\right)\right) \\
& =\exp \left(N f_{\beta, \mu}\left(z_{c}(\beta)\right)\right) \exp \left(\frac{i}{3} w_{1}^{3}\right) \\
\frac{1}{z_{1}^{x_{1}-j+1-\mu N}} & \sim \exp \left(\left(\xi_{1}-\lambda\right)\left(i w_{1}-\tau_{1}\right)\right) .
\end{aligned}
$$

In (4.16), we used (2.27). By using (4.14) - (4.16), and

$$
\exp \left(N f_{\beta, \mu}\left(z_{c}(\beta)\right)\right) \sim(1-\alpha)^{\frac{2(1+\alpha)^{2 / 3} \tau_{1} N^{2 / 3}}{\alpha^{1 / 3}}} \exp \left(\tau_{1}^{3} / 3\right),
$$

we get

$$
D N^{1 / 3} \psi_{1}\left(x_{1}-j\right) \sim(1-\alpha)^{\frac{2(1+\alpha)^{2 / 3} N^{2 / 3} \tau_{1}}{\alpha^{1 / 3}}} \exp \left(\tau_{1}^{3} / 3-\xi_{1} \tau_{1}+\tau_{1} \lambda\right) \times \operatorname{Ai}\left(\xi_{1}-\lambda\right) .
$$

Similarly $\psi_{2}\left(x_{2}\right)$ becomes

$$
D N^{1 / 3} \psi_{2}\left(x_{2}\right) \sim(1-\alpha)^{\frac{-2(1+\alpha)^{2 / 3} N^{2 / 3} \tau_{2}}{\alpha^{1 / 3}}} \exp \left(-\tau_{2}^{3} / 3+\xi_{2} \tau_{2}\right) \times \operatorname{Ai}\left(\xi_{2}\right) .
$$

Here, in the equations above, we used the integral representation of the Airy function

$$
\operatorname{Ai}(x)=\int_{-\infty}^{\infty} d \lambda e^{i x \lambda+\frac{i}{3} \lambda^{3}} .
$$


Next, we also apply the saddle point analysis to $h_{m-j}\left(\alpha^{2 N}\right) / h_{m}\left(\alpha^{2 N}\right)$ in (4.5) under the scaling (2.30) and (4.8) as $N \rightarrow \infty$. From (A.12), (A.19), and (2.30), $h_{m-j}\left(\alpha^{2 N}\right)$ is expressed as

$$
h_{m-j}\left(\alpha^{2 N}\right)=\frac{1}{2 \pi i} \int_{C_{1}} e^{-N g(z)} \frac{d z}{z^{-j+1}},
$$

where the contour $C_{1}$ represents the unit circle surrounding the origin anticlockwise, and

$$
g(z)=2 \log (1-\alpha z)+\left(\frac{2 \alpha}{1-\alpha}+\delta\right) \log z, \quad \delta=\frac{B \omega}{N^{1 / 3}} .
$$

The saddle point $w_{c}$ of $g(z)$ is

$$
w_{c}=\frac{2 \alpha+(1-\alpha) \delta}{2 \alpha+\alpha(1-\alpha) \delta} .
$$

When we deform the path of $z$ to

$$
z=w_{c}\left(1+\frac{1-\alpha}{\alpha^{1 / 2} N^{1 / 2}} i w\right) \sim 1+\frac{\omega}{D N^{1 / 3}}+\frac{1-\alpha}{\alpha^{1 / 2} N^{1 / 2}} i w,
$$

we find

$$
\exp (-N g(z)) \sim \exp \left(-N g\left(w_{c}\right)-w^{2}\right), \frac{1}{z^{-j+1}} \sim e^{\omega \lambda} .
$$

Therefore, the asymptotic form of $h_{m-j}\left(\alpha^{2 N}\right)$ becomes

$$
h_{m-j}\left(\alpha^{2 N}\right) \sim \frac{N^{-1 / 2}}{2 \sqrt{\pi}} \frac{1-\alpha}{\alpha}(1-\alpha)^{-2 N} \exp \left(-\frac{\alpha^{1 / 3} \omega^{2} N^{\frac{1}{3}}}{(1+\alpha)^{2 / 3}}+\omega^{3} / 3+\omega \lambda\right) .
$$

Thus, we get

$$
\frac{h_{m-j}\left(\alpha^{2 N}\right)}{h_{m}\left(\alpha^{2 N}\right)} \sim \exp (\lambda \omega)
$$

From (4.18), (4.19), and (4.27), we obtain

$$
D N^{1 / 3} \sum_{j=1}^{m} \frac{h_{m-j}\left(\alpha^{2 N}\right)}{h_{m}\left(\alpha^{2 N}\right)} \psi_{1}\left(x_{1}-j\right) \psi_{2}\left(x_{2}\right) \sim P \times \int_{0}^{\infty} d \lambda e^{\lambda\left(\tau_{1}+\omega\right)} \operatorname{Ai}\left(\xi_{1}-\lambda\right) \operatorname{Ai}\left(\xi_{2}\right) .
$$

Here the prefactor $P$ is defined in (4.4). From (4.3) and (4.28) and noting that $P$ does not contribute to determinant calculation, we finally obtain the desired expression. However, it is obvious that this expression is valid only for the case $\tau_{i}+\omega<0(i=1,2)$ because in the case $\tau_{i}+\omega>0$, the integration in (4.28) is divergent. 
In order to get the asymptotic form in the latter case, we need another expression for (4.5). By using (A.19), we rewrite the term $\sum_{j=1}^{m} h_{m-j}\left(\alpha^{2 N}\right) z^{-j}$ in (4.5),

$$
\sum_{j=1}^{m} h_{m-j}\left(\alpha^{2 N}\right) z^{j}=\frac{z^{m}}{(1-\alpha / z)^{2 N}}-\sum_{j=-\infty}^{0} h_{m-j}\left(\alpha^{2 N}\right) z^{j}
$$

Thus, we obtain

$$
\sum_{j=1}^{m} \frac{h_{m-j}\left(\alpha^{2 N}\right)}{h_{m}\left(\alpha^{2 N}\right)} \psi_{1}\left(x_{1}-j\right) \psi_{2}\left(x_{2}\right)=\frac{1}{h_{m}\left(\alpha^{2 N}\right)} \varphi\left(x_{1}\right) \psi_{2}\left(x_{2}\right)-\sum_{j=-\infty}^{0} \frac{h_{m-j}\left(\alpha^{2 N}\right)}{h_{m}\left(\alpha^{2 N}\right)} \psi_{1}\left(x_{1}-j\right) \psi_{2}\left(x_{2}\right),
$$

where

$$
\varphi\left(x_{1}\right)=\frac{1}{2 \pi i} \int_{C_{1}} \frac{d z_{1}}{z_{1}^{1+x_{1}-m}}\left(1-\alpha / z_{1}\right)^{u_{1}-2 N}\left(1-\alpha z_{1}\right)^{u_{1}-2 N},
$$

and $\psi_{1}(x)$ (resp. $\left.\psi_{2}(x)\right)$ is defined in (4.6) (resp. (4.7)).

We can easily get the asymptotic forms of $\varphi(x)$ by using the saddle point analysis in a manner similar to the derivation of (4.26). The result is

$$
\begin{aligned}
& \varphi\left(x_{1}\right) \\
& \sim \frac{N^{-1 / 2}}{2 \pi^{1 / 2}} \frac{1-\alpha}{\alpha}(1-2 N)^{-2 N}(1-\alpha)^{\frac{2(1+\alpha)^{2 / 3} \tau_{1} N^{2 / 3}}{\alpha^{1 / 3}}} \exp \left(-\frac{\alpha^{1 / 3} \omega^{2} N^{1 / 3}}{(1+\alpha)^{2 / 3}}-\tau_{1}^{2} \omega-\tau_{1} \omega^{2}+\xi_{1} \omega\right) .
\end{aligned}
$$

From (4.19), (4.26), and (4.32), we have

$$
D N^{1 / 3} \frac{\varphi\left(x_{1}\right)}{h_{m}\left(\alpha^{2 N}\right)} \psi_{2}\left(x_{2}\right) \sim P \times e^{-\left(\omega+\tau_{1}\right)^{3} / 3+\xi_{1}\left(\omega+\tau_{1}\right)} \operatorname{Ai}\left(\xi_{2}\right) .
$$

Analogous to the derivation of (4.28), we also have

$$
-D N^{1 / 3} \sum_{j=-\infty}^{0} \frac{h_{m-j}\left(\alpha^{2 N}\right)}{h_{m}\left(\alpha^{2 N}\right)} \psi_{1}\left(x_{1}-j\right) \psi_{2}\left(x_{2}\right) \sim-P \times \int_{0}^{\infty} d \lambda e^{-\lambda\left(\tau_{1}+\omega\right)} \operatorname{Ai}\left(\xi_{1}+\lambda\right) \operatorname{Ai}\left(\xi_{2}\right) .
$$

From these two relations, we get the desired expression also in the case $\tau_{i}+\omega>0$.

\section{Conclusion}

In this paper, we have studied the correlation function (2.4) of the process (1.2). This process is the generalization of the Schur process (1.1) [7] in the sense that in the picture of nonintersecting random walk, the walkers end at fixed sites, as depicted in Fig. 2. At first, we have obtained the integral representation of the correlation kernel (Theorem 2.1). 
The result is the generalization of the one in the Schur process obtained in 17, 14, 23]. The derivation is given in Sec. 3. The key technique is the calculation of the inverse of the matrix $\mathcal{A}(2.9)$ with semi-infinite rank. This technique can be regarded as the generalization of the one discussed in [14]. Next, by using the integral representation, we have obtained the edge scaling limit of the correlation kernel in the special case (2.24) and (2.25). The result is summarized as Theorem 2.2 and the proof is given in Sec. 4. We have found that the limiting correlation kernel is equivalent to the one obtained in other determinantal processes such as the random matrix with external source, the PNG model, and statistics.

We list the future problems as follows.

1. In this paper, we have discussed the asymptotics of the correlation kernel only in the simple case (2.24) and (2.25), although the process (1.2) has many parameters $\mu^{(4 N)}$ and $\left\{a^{(i)}\right\}_{i=1, \cdots, 4 N}$. It would be interesting to investigate the limiting behavior of the correlation kernel in a more general situation and how it depends on these parameters.

2. The correlation kernel may be closely related to the orthogonal polynomials. In the Schur process, the correlation kernel (the first term in (2.18) ) can be represented in terms of the Meixner polynomial in the single time case [42]. On the other hand, it has been recently revealed that the correlation kernel corresponding to the random Hermitian matrix with external source can be expressed in terms of the multiple orthogonal polynomials 43 . In the discretized process (1.2), is the correlation kernel related to any discrete analog of the multiple orthogonal polynomial?

3. In Theorem 2.1, the second term in (2.18), which is due to the partition $\mu^{(4 N)}$ in (1.2), can be expressed by using the Schur function. This fact raises our hope that there exists some deep connection between the process (1.2) and the theory of integrable system and representation theory. In particular, it is interesting to view the problem in perspective of the Kadomtsev-Petviashvili and Toda Lattice hierarchies [44, 45]. Recently, the relationship between the stochastic processes such as the random turn walk and soliton theory has been discussed in [45]. This approach may be useful for studying this topic.

\section{A Vertex operators and symmetric functions}

Let $c_{j}^{\dagger}$ and $c_{i}(i, j \in \mathbb{Z}=(\cdots,-1,0,1, \cdots))$ be the creation and annihilation operators that satisfy the fermion anticommutation relation

$$
\begin{aligned}
& \left\{c_{i}, c_{j}^{\dagger}\right\}:=c_{i} c_{j}^{\dagger}+c_{j}^{\dagger} c_{i}=\delta_{i j}, \\
& \left\{c_{i}, c_{j}\right\}=\left\{c_{i}^{\dagger}, c_{j}^{\dagger}\right\}=0 .
\end{aligned}
$$

One particle state $|i\rangle$ is defined as

$$
|i\rangle=c_{i}^{\dagger}|\Omega\rangle
$$


where $|\Omega\rangle$ is the vacuum state without particle.

The mode operators $c(z), c^{\dagger}(z)$ and the vertex operators $H_{ \pm}(s)$, where $s=\left(s_{1}, s_{2}, \cdots\right)$, are defined as follows:

$$
\begin{aligned}
& c^{\dagger}(z)=\sum_{i=-\infty}^{\infty} z^{i} c_{i}^{\dagger}, \quad c(z)=\sum_{i=-\infty}^{\infty} z^{-i} c_{i}, \\
& H_{ \pm}(s)=\exp \left(\sum_{n=1}^{\infty} s_{n} \beta_{ \pm n}\right), \text { where } \beta_{ \pm n}=\sum_{k=-\infty}^{\infty} c_{k \pm n}^{\dagger} c_{k}, \quad(n=1,2, \cdots) .
\end{aligned}
$$

We summarize the basic properties of these operators, which are used for our discussion,

$$
\begin{aligned}
& H_{ \pm}(s)|\Omega\rangle=|\Omega\rangle, \\
& H_{ \pm}(s) c^{\dagger}(z)=\gamma\left(z^{\mp 1}, s\right) c^{\dagger}(z) H_{ \pm}(s), \\
& H_{ \pm}(s) c(z)=\gamma\left(z^{\mp 1}, s\right)^{-1} c(z) H_{ \pm}(s), \text { where } \gamma(z, s)=\exp \left(\sum_{n=1}^{\infty} s_{n} z^{n}\right), \\
& H_{+}(s) H_{-}\left(s^{\prime}\right) \stackrel{\langle j|\cdot| \cdot\rangle}{=} H_{-}\left(s^{\prime}\right) H_{+}(s),
\end{aligned}
$$

In $(\underline{A .9})$, the symbol $\stackrel{\langle j|\cdot| i\rangle}{=}$ means an equality on one particle space. Note that (A.9) is different from the ordinary one. As discussed in [7] when the product $H_{+}(s) H_{-}\left(s^{\prime}\right)$ appears in another vacuum state where infinitely many particles are occupied up to the origin, the following relation holds:

$$
H_{+}(s) H_{-}\left(s^{\prime}\right)=e^{\sum_{n=1}^{\infty} s_{n} s_{n}^{\prime}} H_{-}\left(s^{\prime}\right) H_{+}(s) .
$$

When we set the variable $s$ of the vertex operators,

$$
s_{j}=\frac{1}{j} \sum_{i=1}^{n} a_{i}^{j},
$$

we find that $\gamma(z, s)$ in (A.8) is given as the generating function of the complete symmetric function $h_{j}(a)$,

$$
\gamma(z, s)=\prod_{i=1}^{n} \frac{1}{1-a_{i} z}=\sum_{j} h_{j}(a) z^{j} .
$$

By using this and the properties (A.6) $-\left(\right.$ A.8), we can describe $h_{j}(a)$ as

$$
h_{j-i}(a)=\left\langle j\left|H_{+}(s)\right| i\right\rangle=\left\langle i\left|H_{-}(s)\right| j\right\rangle,
$$

under the parameterization (A.11).

We also often use the following functions:

$$
\begin{aligned}
& c_{j-k}(v)=\left\langle j\left|\Lambda_{+}(v)\right| k\right\rangle=\left\langle k\left|\Lambda_{-}(v)\right| j\right\rangle, \\
& d_{j-k}(v)=\left\langle j\left|\Lambda_{+}^{-1}(v)\right| k\right\rangle=\left\langle k\left|\Lambda_{-}^{-1}(v)\right| j\right\rangle,
\end{aligned}
$$


where

$$
\begin{aligned}
& \Lambda_{ \pm}(v)=\prod_{j=1}^{2 N} H_{ \pm}\left(v^{(j)}\right) \\
& v=\left(v^{(1)}, v^{(2)}, \cdots, v^{(2 N)}\right) .
\end{aligned}
$$

The properties that we often use are summarized as follows:

$$
\begin{aligned}
& \sum_{i=0}^{k} c_{i}(v) d_{k-i}(v)= \begin{cases}1, & \text { for } k=0, \\
0, & \text { for } 1 \leq k\end{cases} \\
& \sum_{i=0}^{\infty} c_{i}(v) z^{i}=\prod_{i=1}^{2 N} \gamma\left(z, v^{(i)}\right), \quad \sum_{i=0}^{\infty} d_{i}(v) z^{i}=\prod_{i=1}^{2 N} \gamma^{-1}\left(z, v^{(i)}\right) .
\end{aligned}
$$

\section{B Proof of (3.26)}

In this appendix, we derive (3.26) by deforming the right hand side of (3.25). By noticing

$$
\mathcal{A}_{i j}^{\prime}=\sum_{k=1}^{\infty} d_{j-k}\left(s^{\prime}\right) d_{x_{k}-x_{i}}(s)
$$

and (A.19), we rewrite the first term of $(3.25)$ as

$$
\begin{aligned}
\sum_{i=n+1}^{\infty} \sum_{j=1}^{\infty} z_{1}^{-i} \mathcal{A}_{i j}^{\prime} z_{2}^{j} & =\sum_{k=1}^{\infty} z_{2}^{k} \prod_{j=1}^{2 N} \gamma^{-1}\left(z_{2}, s^{(2 j)}\right) \sum_{i=n+1}^{\infty} d_{x_{k}+i-1}(s) z_{1}^{-i} \\
& =\sum_{k=n+1}^{\infty}\left(\frac{z_{2}}{z_{1}}\right)^{k} \prod_{j=1}^{2 N} \gamma^{-1}\left(z_{1}^{-1}, s^{(2 j-1)}\right) \gamma^{-1}\left(z_{2}, s^{(2 j)}\right) \\
& +\sum_{k=1}^{n} z_{2}^{k} \sum_{i=n+1}^{\infty} d_{x_{k}+i-1}(s) z_{1}^{-i} \prod_{j=1}^{2 N} \gamma^{-1}\left(z_{2}, s^{(2 j)}\right)
\end{aligned}
$$

We need a quite lengthy calculation for the second term of (3.25). At first, by using (3.18) and (3.23), the part $\sum_{i=n+1}^{\infty} z^{-i} b_{i k}$ in the term can be represented as

$$
\begin{aligned}
\sum_{i=n+1}^{\infty} z_{1}^{-i} b_{i k} & =-\sum_{j=1}^{n} b_{j k} \sum_{i=n+1}^{\infty} a_{i j} z_{1}^{-i} \\
& =-\sum_{j=1}^{n} b_{j k} \sum_{i=n+1}^{\infty}\left(\sum_{\ell=0}^{i-n-1} c_{m_{j}+i-1-\ell}(s) d_{\ell}(s)+\sum_{a=1}^{n} c_{m_{j}+a-1}(s) d_{m_{a}+i-1}(s)\right) z_{1}^{-i} .
\end{aligned}
$$


In this equation, we notice that the term $\sum_{i=n+1}^{\infty} \sum_{\ell=0}^{i-n-1} c_{m_{j}+i-1-\ell}(s) d_{\ell}(s) z_{1}^{-i}$ becomes

$$
\sum_{i=n+1}^{\infty} \sum_{\ell=0}^{i-n-1} c_{m_{j}+i-1-\ell}(s) d_{\ell}(s) z_{1}^{-i}=-\sum_{\ell=0}^{m_{j}+n-1} c_{m_{j}+n-1-\ell}(s) z_{1}^{\ell-n} \prod_{i=1}^{2 N} \gamma^{-1}\left(z_{1}^{-1}, s^{(2 i-1)}\right)+z_{1}^{m_{j}-1} .
$$

The derivation is given as follows. By noticing (A.18) and (A.19), one gets

$$
\begin{aligned}
& \sum_{i=n+1}^{\infty} \sum_{\ell=0}^{i-n-1} c_{m_{j}+i-1-\ell}(s) d_{\ell}(s) z_{1}^{-i} \\
= & -\sum_{i=n+1}^{\infty} \sum_{\ell=i-n}^{m_{j}+i-1} c_{m_{j}+i-1-\ell}(s) d_{\ell}(s) z_{1}^{-i}=-\sum_{i=n+1}^{\infty} \sum_{\ell^{\prime}=0}^{m_{j}+n-1} c_{m_{j}+n-1-\ell^{\prime}}(s) d_{\ell^{\prime}+i-n}(s) z_{1}^{-i} \\
= & -\sum_{\ell^{\prime}=0}^{m_{j}+n-1} c_{m_{j}+n-1-\ell^{\prime}}(s) z_{1}^{\ell^{\prime}-n}\left(\sum_{i=n+1}^{\infty} d_{\ell^{\prime}+i-n}(s) z_{1}^{-i-\ell^{\prime}+n}\right) \\
= & -\sum_{\ell^{\prime}=0}^{m_{j}+n-1} c_{m_{j}+n-1-\ell^{\prime}}(s) z_{1}^{\ell^{\prime}-n}\left(\prod_{i=1}^{2 N} \gamma^{-1}\left(z_{1}^{-1}, s^{(2 i-1)}\right)-\sum_{i=0}^{\ell^{\prime}} d_{i}(s) z_{1}^{-i}\right)
\end{aligned}
$$

By noting again (A.18), we find

$$
\sum_{\ell^{\prime}=0}^{m_{j}+n-1} c_{m_{j}+n-1-\ell^{\prime}}(s) \sum_{i=0}^{\ell^{\prime}} d_{i}(s) z_{1}^{\ell^{\prime}-i-n}=z_{1}^{m_{j}-1} .
$$

Then, we eventually obtain (B.4). On the other hand, from $(\overline{B .1})$, the other term $\sum_{i=1}^{\infty} \mathcal{A}_{k j}^{\prime} z_{2}^{j}$ in the second term in (3.25) is

$$
\sum_{j=1}^{\infty} \mathcal{A}_{k j}^{\prime} z_{2}^{j}=\sum_{b=1}^{k} d_{m_{b}-m_{k}}(s) z_{2}^{b} \prod_{i=1}^{2 N} \gamma^{-1}\left(z_{2}, s^{(2 i)}\right)
$$

Thus, from (B.3), (B.4), and (B.7), we get

$$
\begin{aligned}
& \frac{1}{b_{0}} \sum_{k=1}^{n} \sum_{i=n+1}^{\infty} z_{1}^{-i} b_{i k} \sum_{j=1}^{\infty} \mathcal{A}_{k j}^{\prime} z_{2}^{j} \\
& =-\frac{1}{b_{0}} \sum_{k=1}^{n} \sum_{j=1}^{n} b_{j k} z_{1}^{m_{j}-1} \sum_{c=1}^{\infty} \mathcal{A}_{k c}^{\prime} z_{2}^{c} \\
& -\frac{1}{b_{0}} \sum_{k=1}^{n} \sum_{j=1}^{n} b_{j k} \sum_{i=n+1}^{\infty} \sum_{a=1}^{n} c_{m_{j}+a-1}(s) d_{m_{a}+i-1}(s) z_{1}^{-i} \sum_{b=1}^{k} d_{m_{b}-m_{k}}(s) z_{2}^{b} \prod_{i=1}^{2 N} \gamma^{-1}\left(z_{2}, s^{(2 i)}\right) \\
& +\frac{1}{b_{0}} \sum_{k=1}^{n} \sum_{j=1}^{n}\left(b_{j k} \sum_{\ell=0}^{m_{j}+n-1} c_{m_{j}+n-1}(s) z_{1}^{\ell-n} \sum_{b=1}^{k} d_{m_{b}-m_{k}}(s) z_{2}^{b}\right) \prod_{i=1}^{2 N} \gamma^{-1}\left(z_{1}^{-1}, s^{(2 i-1)}\right) \gamma^{-1}\left(z_{2}, s^{(2 i)}\right) .
\end{aligned}
$$


Note that the first term in this equation and the last one in (3.25) cancel each other. We can also find that the second term in (B.8) cancels out the second one in $(\bar{B} .2)$ by the following discussion. By using the properties

$$
\begin{aligned}
& \sum_{k=1}^{n} b_{j k} d_{m_{b}-m_{k}}(s)=\tilde{\mathcal{C}}_{(b j)}, \\
& \sum_{j=1}^{n} c_{m_{j}+a-1}(s) \tilde{\mathcal{C}}_{(b j)}=\delta_{a b} \operatorname{det} \mathcal{C}=\delta_{a b} b_{0},
\end{aligned}
$$

where $\tilde{\mathcal{C}}_{(b j)}$ is the $b j$ cofactor of the matrix $\mathcal{C}$ (3.21), we have

$$
\begin{aligned}
& -\frac{1}{b_{0}} \sum_{k=1}^{n} \sum_{j=1}^{n} b_{j k} \sum_{i=n+1}^{\infty} \sum_{a=1}^{n} c_{m_{j}+a-1}(s) d_{m_{a}+i-1}(s) z_{1}^{-i} \sum_{b=1}^{k} d_{m_{b}-m_{k}}(s) z_{2}^{b} \prod_{i=1}^{2 N} \gamma^{-1}\left(z_{2}, s^{(2 i)}\right) \\
& =-\sum_{a=1}^{n} z_{2}^{a} \sum_{i=n+1}^{\infty} d_{m_{a}+i-1}(s) z_{1}^{-i} \prod_{i=1}^{2 N} \gamma^{-1}\left(z_{2}, s^{(2 i)}\right) .
\end{aligned}
$$

Furthermore, from relations $(\underline{B .9})$ and $(\underline{B .10})$, we can also rewrite the third term in (B.8) as

$$
\begin{aligned}
& \frac{1}{b_{0}} \sum_{k=1}^{n} \sum_{j=1}^{n}\left(b_{j k} \sum_{\ell=0}^{m_{j}+n-1} c_{m_{j}+n-1-\ell}(s) z_{1}^{\ell-n} \sum_{b=1}^{k} d_{m_{b}-m_{k}}(s) z_{2}^{b}\right) \prod_{i=1}^{2 N} \gamma^{-1}\left(z_{1}^{-1}, s^{(2 i-1)}\right) \gamma^{-1}\left(z_{2}, s^{(2 i)}\right) \\
& =\left(\frac{1}{b_{0}} \sum_{j=1}^{n} \sum_{\ell^{\prime}=0}^{m_{j}-1} c_{\ell^{\prime}}(s) z_{1}^{m_{j}-1-\ell^{\prime}} \sum_{b=1}^{n} \tilde{\mathcal{C}}_{(b j)} z_{2}^{b}+\sum_{b=1}^{n}\left(\frac{z_{2}}{z_{1}}\right)^{b}\right) \\
& \times \prod_{i=1}^{2 N} \gamma^{-1}\left(z_{1}^{-1}, s^{(2 i-1)}\right) \gamma^{-1}\left(z_{2}, s^{(2 i)}\right) .
\end{aligned}
$$

From $(\underline{\mathrm{B} .8}),(\underline{\mathrm{B} .11})$, and $(\underline{\mathrm{B} .12})$, we get

$$
\begin{aligned}
& \frac{1}{b_{0}} \sum_{k=1}^{n} \sum_{i=n+1}^{\infty} z_{1}^{-i} b_{i k} \sum_{j=1}^{\infty} \mathcal{A}_{k j}^{\prime} z_{2}^{j} \\
& =-\frac{1}{b_{0}} \sum_{k=1}^{n} \sum_{j=1}^{n} b_{j k} z_{1}^{m_{j}-1} \sum_{c=1}^{\infty} \mathcal{A}_{k c}^{\prime} z_{2}^{c}-\sum_{a=1}^{n} z_{2}^{a} \sum_{i=n+1}^{\infty} d_{m_{a}+i-1}(s) z_{1}^{-i} \prod_{i=1}^{2 N} \gamma^{-1}\left(z_{2}, s^{(2 i)}\right) \\
& +\left(\frac{1}{b_{0}} \sum_{j=1}^{n} \sum_{\ell^{\prime}=0}^{m_{j}-1} c_{\ell^{\prime}}(s) z_{1}^{m_{j}-1-\ell^{\prime}} \sum_{b=1}^{n} \tilde{\mathcal{C}}_{(b j)} z_{2}^{b}+\sum_{b=1}^{n}\left(\frac{z_{2}}{z_{1}}\right)^{b}\right) \\
& \quad \times \prod_{i=1}^{2 N} \gamma^{-1}\left(z_{1}^{-1}, s^{(2 i-1)}\right) \gamma^{-1}\left(z_{2}, s^{(2 i)}\right) .
\end{aligned}
$$

Hence, from (3.25), (B.2), and (B.13), we finally obtain (3.26). 


\section{Acknowledgments}

The work of T.I. is supported by the Core Research for Evolutional Science and Technology of Japan Science and Technology Agency. The work of T.S. is supported by the Grantin-Aid for Young Scientists (B), the Ministry of Education, Culture, Sports, Science and Technology, Japan.

\section{References}

[1] M. Kardar, G. Parisi, and Y. C. Zhang, Dynamic scaling of growing interfaces, Phys. Rev. Lett., 56: 889-892, 1986.

[2] K. Johansson, Random matrices and determinantal processes, In A. Bovier, F. Dunlop, A. van Enter, F. den Hollander, and J. Dalibard eds, Mathematical Statistical Physics : Lecture Notes of the Les Houches Summer School, Session LXXXIII, 1-56, Elsevier, 2006, math-ph/0510038.

[3] H. Spohn, Exact solutions for KPZ-type growth processes, random matrices, and equilibrium shapes of crystals, Physica. A., 369: 71-99, 2006.

[4] P. L. Ferrari and M. Prähofer, One-dimensional stochastic growth and Gaussian ensembles of random matrices, Markov Processes Relat. Fields, 12: 203-234, 2006.

[5] S. N. Majumdar, Random matrices, the Ulam Problem, directed polymers \& growth models, and sequence matching, In J. P. Bouchaud, M. Mezard, and J. Dalibard eds, Complex Systems: Lecture Notes of the Les Houches Summer School, Session $L X X X V, 179-216$, Elsevier, 2007, cond-mat/0701193.

[6] T. Sasamoto, Fluctuations of the one-dimensional asymmetric exclusion process using random matrix techniques, J. Stat. Mech., P07007, 2007.

[7] A. Okounkov and N. Reshetikhin, Correlation function of Schur process with application to local geometry of a random 3-dimensional Young diagram, J. Amer. Math. Soc., 16: 581-603, 2003.

[8] A. J. Guttmann, A. L. Owczarek, and X. G. Viennot, Vicious walkers and Young tableaux I: without walls, J. Phys. A, 31: 8123-8135, 1998.

[9] J. Baik, Random vicious walks and random matrices, Com. Pure Appl. Math., 53: 1385-1410, 2000.

[10] P. J. Forrester, Random walks and random permutations, J. Phys. A, 34: L417-L423, 2000.

[11] T. Nagao and P. J. Forrester Vicious random walkers and a discretization of gaussian random matrix ensembles Nuc. Phys. B, 620: 551-565, 2002. 
[12] M. Katori and H. Tanemura, Scaling limit of vicious walks and two-matrix model, Phys. Rev. E, 66: 011105, 2002.

[13] M. Katori and H. Tanemura, Nonintersecting Paths, Noncolliding Diffusion Processes and Representation Theory, RIMS kokyuroku, 1438: 83-102, 2005, math/0501218.

[14] K. Johansson, Discrete polynuclear growth and determinantal processes, Commun. Math. Phys., 242: 277-329, 2003.

[15] P. L. Ferrari and H. Spohn, Step fluctuations for a faceted crystal, J. Stat. Phys., 113: 1-46, 2003.

[16] K. Johansson, The arctic circle boundary and the Airy process, Ann. Prob., 33: 1-30, 2005.

[17] T. Imamura and T. Sasamoto, Dynamics of a tagged particle in the asymmetric exclusion process with the step initial condition, J. Stat. Phys., 128: 799-846, 2007.

[18] M. Prähofer and H. Spohn, Scale invariance of the PNG droplet and the Airy process, J. Stat. Phys., 108: 1071-1106, 2002.

[19] J. Baik and E. M. Rains, Limiting distributions for a polynuclear growth model with external sources, J. Stat. Phys., 100: 523-542, 2000.

[20] T. Imamura and T. Sasamoto, Fluctuations of the one-dimensional polynuclear growth model with external sources, Nucl. Phys. B, 699: 503-544, 2004.

[21] T. Imamura and T. Sasamoto, Polynuclear growth model with external source and random matrix model with deterministic source, Phys. Rev. E, 71: 041606, 2005.

[22] A. Okounkov, Infinite wedge and random partitions, Selecta Math. (New Ser.), 7: 57-81, 2001.

[23] A. Borodin and E. M. Rains, Eynard-Mehta theorem, Schur process, and their Pfaffian analogs, J. Stat. Phys., 121: 291-317, 2006.

[24] M. Katori and H. Tanemura, Symmetry of matrix-valued stochastic processes and noncolliding diffusion particle systems, J. Math. Phys., 45: 3058-3085, 2004.

[25] M. Katori and H. Tanemura, Noncolliding Brownian motion and determinantal processes, J. Stat. Phys., 129, 1233-1277, 2007.

[26] C. A. Tracy and H. Widom, Differential equations for Dyson processes, Commun. Math. Phys., 252: 7-41, 2004.

[27] C. A. Tracy and H. Widom, Nonintersecting Brownian excursions, Ann. Appl. Prob., 17: 953-979, 2007. 
[28] A. I. Aptekarev, P. M. Bleher and A. B. J. Kuijlaars, Large $n$ limit of Gaussian random matrices with external source, part II, Commun. Math. Phys., 259: 367-389, 2005.

[29] M. Adler, J. Delepine, and P. van Moerbeke, Dyson's non-intersecting Brownian motions with a few outliers, arXiv:0707.0442.

[30] B. Eynard and M. L. Mehta, Matrices coupled in a chain: I. Eigenvalue correlations, J. Phys. A, 31: 4449-4456, 1998.

[31] C. A. Tracy and H. Widom, Correlation functions, cluster functions, and spacing distributions for random matrices, J. Stat. Phys., 92: 809-835, 1998.

[32] B. Lindström, On the vector representations of induced matroids, Bull. London Math. Soc., 5: 85-90, 1973.

[33] I. Gessel and G. Viennot, Binomial determinants, paths, and hook length formulae, Adv. Math., 58: 300-321, 1985.

[34] S. Karlin and L. McGregor, Coincidence probabilities, Pacific J. Math., 9: 1141-1164, 1959.

[35] R. P. Stanley, Enumerative Combinatorics Volume 2, Cambridge University Press, 1999.

[36] P. J. Forrester, The spectrum edge of random-matrix ensemble, Nucl. Phys. B, 402: 709-728, 1993.

[37] C. A. Tracy and H. Widom, Level-spacing distributions and the Airy kernel, Commun. Math. Phys., 159: 151-174, 1994.

[38] A. M. S. Macêdo, Universal parametric correlations at the soft edge of spectrum of random matrix ensembles, Europhys. Lett., 26: 641-646, 1994.

[39] P. J. Forrester, T. Nagao, and G. Honner, Correlations for the orthogonal-unitary and symplectic transitions at the hard and soft edges, Nucl. Phys. B, 553: 601-643, 1999.

[40] P. J. Forrester, Painlevé transcendent evaluation of the scaled distribution of the smallest eigenvalue in the Laguerre orthogonal and symplectic ensembles, nlin.SI/0005064.

[41] J. Baik, G. Ben Arous, and S. Péché, Phase transition of the largest eigenvalue for non-null complex sample covariance matrices, Ann. Prob., 33: 1643-1697, 2005.

[42] K. Johansson, Shape fluctuations and random matrices, Commun. Math. Phys., 209: 437-476, 2000.

[43] P. M. Bleher and A. B. J. Kuijlaars. Random matrices with external source and multiple orthogonal polynomials. Int. Math. Res. Not., 109-129, 2004. 
[44] M. Adler and P. van Moerbeke. Integrals over classical groups, random permutations, Toda and Toeplitz lattices, Com. Pure Appl. Math., 54, 153-205, 2001.

[45] J. Harnad and A. Yu. Orlov. Fermionic construction of tau functions and random processes, Physica D, 235, 168-206, 2007. 


\section{Figure Captions}

Fig. 1: The random walk interpretation of the weight (1.1). Note that the $i$ th walker starts at the point $-i+1$ and returns to the initial point at the end by way of the position $\lambda_{i}^{(n)}-i+1$ at time $n$. In this example, the partitions $\lambda^{(n)}(n=1,2,3)$ are $\lambda^{(1)}=(4,4,2), \lambda^{(2)}=(2,1,1)$, and $\lambda^{(3)}=(4,4,3,2,1)$, respectively.

Fig. 2: The random walk interpretation of the weight (1.2). In this case, the $i$ th walker starts at the point $1-i$ and ends at the point $\mu_{i}^{(4 N)}-i+1$, which is arbitrary but fixed. 

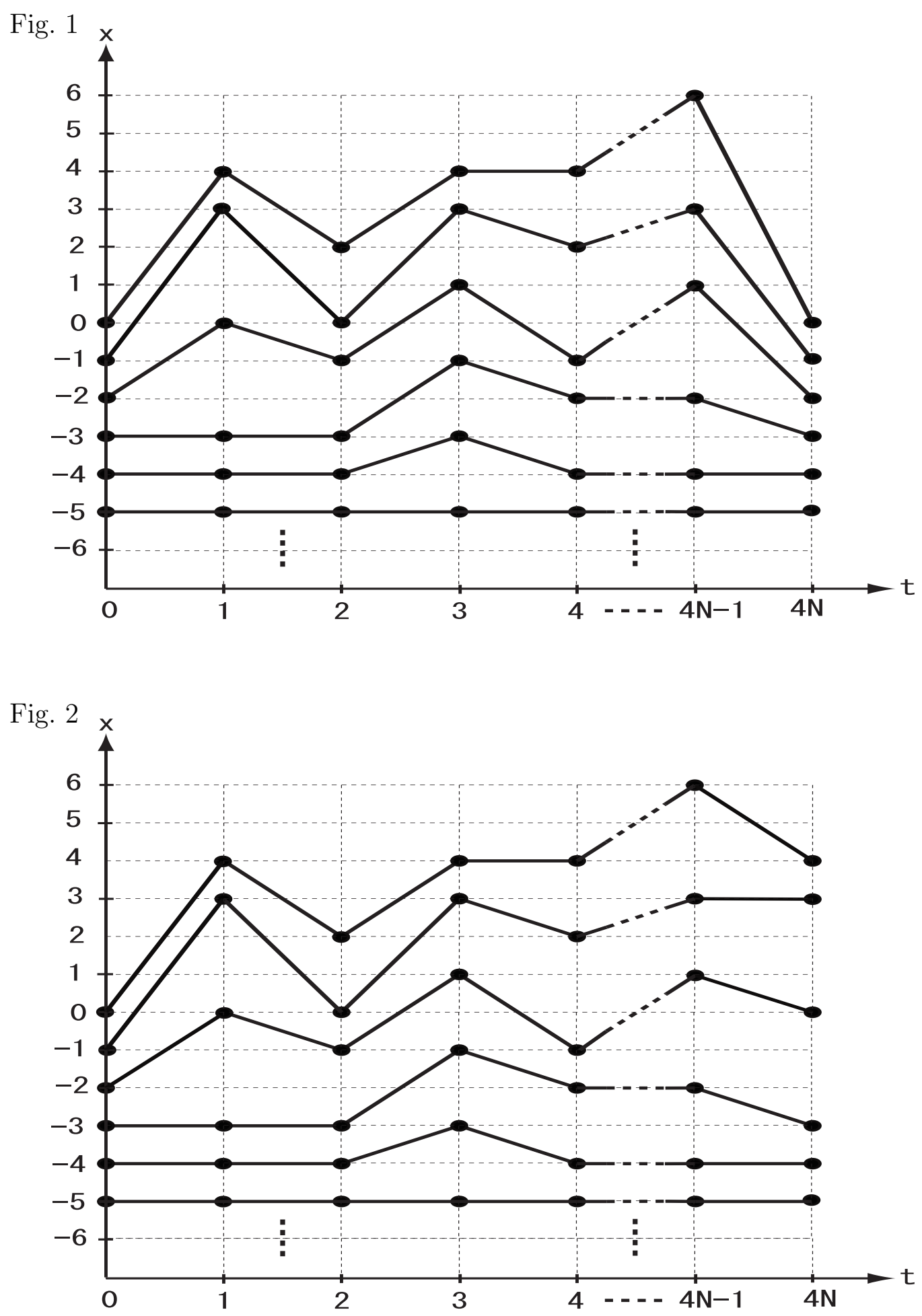